Pacific Journal of Mathematics

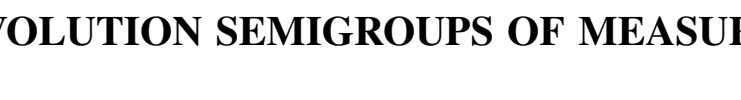




\section{CONVOLUTION SEMIGROUPS OF MEASURES}

\section{IRVING GLICKSBERG}

Let $S$ be a compact topological semigroup, $C(S)$ the Banach space of all continuous complex valued functions on $S$, and $\widetilde{S}$ the normalized nonnegative regular Borel measures on $S$. Under convolution and the $\omega^{*}$ topology of $C(S)^{*}, \tilde{S}$ and the unit ball $\widetilde{\tilde{S}}$ of $C(S)^{*}$ each form a compact semigroup. The main purpose of this paper is the determination of all subgroups of $\tilde{S}$ and $\tilde{\tilde{S}}$ when $S$ is abelian.

In the case in which $S$ is a group, J. G. Wendel [10] has determined the idempotents in $\tilde{S}$ : they are just the Haar measures of subgroups of $S$. This fails to hold for the general compact semigroup $S$, but does remain valid for compact abelian semigroups, due primarily to the fact that the least ideal in a compact abelian semigroup is a group. Indeed it is just this feature of the abelian case which allows one to complete the one point in Wendel's argument where essential use is made of a group structure, rather than a semigroup structure, for $S$, and further allows one to determine the subgroups of $\tilde{S}$.

The structure of the subgroups of $\widetilde{S}$ (when $S$ is abelian or a group) is quite simple: each subgroup $\Gamma$ of $\tilde{S}$ consists of the $G$ - translates of Haar measure on $g$, where $G$ is a subgroup of $S$, and $g$ a normal subgroup of $G$. Thus $\Gamma$ is just the set of point masses on $G / g$ imbedded in $\widetilde{S}$ in the natural fashion, and we arrive essentially at the fact that the only subgroups of $\tilde{S}$ are the obvious ones. But a consequence of this knowledge is an extension of the Weyl equidistribution theorem: for $\mu$ in $\tilde{S}, N^{-1} \sum_{n=1}^{N} \mu^{n} \underset{\omega^{*}}{\longrightarrow}$ Haar measure of the least ideal of the subsemigroup of $S$ generated by the carrier of $\mu$ (in the group situation this is convergence to Haar measure of the subgroup generated by the carrier).

Finally, in the abelian case, the determination of the subgroups of $\tilde{\tilde{S}}$ is obtained as a consequence; by virtue of a theorem of Eberlein [3] we can apply our results to obtain the subgroups of the convolution semigroup formed by the unit ball of $C_{0}(\mathscr{G})^{*}$ where $\mathscr{G}$ is a locally compact abelian group.

It is a pleasure to record the author's indebtedness to K. de Leeuw for his stimulating comments and suggestions, which were directly

Received July 14, 1958. Presented to the American Mathematical Society, June 21, 1958. 
responsible for many of the results; our indebtedness to Wendel's paper will be self-evident.

1. Preliminaries. We begin with a résumé of the facts and notation we shall use in connection with semigroups, ideals, measures and convolution; for standard results on measure theory and topological groups the reader is referred to $[5,6,9]$. Let $S$ be henceforth a compact semigroup, i.e. a compact (Hausdorff) space with a jointly continuous (binary) operation (multiplication) under which it forms a semigroup. By a subsemigroup of $S$ we shall implicitly mean a closed subsemigroup; a not necessarily closed one will be called an algebraic subsemigroup. By a subgroup $G$ of $S$ we shall mean a (closed) subsemigroup which algebraically forms a group under our operation ; since $G$ is compact, inversion (as is easily seen) is automatically continuous and $G$ is a compact topological group.

(1.1) Suppose now that $S$ is abelian. An ideal $I$ of $S$ is a nonvoid subset closed under multiplication from outside $(S I \subset I)$, and a consequence of compactness is the fact that $S$ contains a least ideal $I=$ $\bigcap_{x \in S} x S$; for $x y S \subset x S \cap y S$ implies $\{x S: x \in S\}$ has the finite intersection property while $x S$ is trivially closed so that $I \neq \phi$. And clearly $I$ is a (closed) ideal contained in any other ideal. Moreover

\section{(1.11) if $E$ is dense in $S$, then $I=\bigcap_{x \in E} x S$.}

For given an open set $V$ containing $I$ we have an $x$ in $S$ with $x S \subset V$ (otherwise the filter generated by $\{x S: x$ in $S\}$ has each of its elements meeting the compact complement $V^{\prime}$ of $V$, whence $\left.I \cap V^{\prime} \neq \phi\right)$. Thus by compactness and the continuity of multiplication we have a $y$ in $E$ near $x$ for which $y S \subset V, I \subset \bigcap_{y \in E} y S \subset V$, and (1.11) follows. Further $I$ is a subgroup of $S$ as well [8]: for $x \in S \Rightarrow x I$ is an ideal contained in $I$, so $x I=I$. Thus if $x \in I$ we have an $e$ in $I$ for which $x e=x$, whence $y x e=y x$; since $I x=x I=I$, $e$ is clearly an identity for $I$. On the other hand $y I=I$ implies that there is a $z$ in $I$ with $y z=e$, and $I$ is a group.

For a non-abelian $S$ we have the usual variety of ideals and the above facts are of course invalid; however it will be convenient to note that if $S$ is a group any sort of ideal must coincide with the full group $S$, and all of our remarks retain their full force.

(1.2) With $S$ abelian or not the fact that $S$ is compact allows us to identify $C(S)^{*}$ with the space of (integrals with respect to) complex regular Borel measures of $S$. We shall use the same letter to denote the functional and the measure, writing $\mu(f)=\int f(x) \mu(d x)$. The norm 
$\|\mu\|$ of $\mu$ in $C(S)^{*}$ is of course its total variation, and the unit ball of $C(S)^{*}, \tilde{\tilde{S}}=\{\mu:\|\mu\| \leqq 1\}$ is compact in the $\omega^{*}$ topology, as is its subspace $\tilde{S}=\{\mu: \mu \geqq 0,\|\mu\|=1\}$.

For $f \in C(S)$ let $f_{x}(y)=f(x y), f^{x}(y)=f(y x)$, so that $f_{x}$ and $f^{x}$ are in $C(S)$. The compactness of $S$ and the continuity of multiplication combine to yield the maps $x \rightarrow f_{x}, x \rightarrow f^{x}$ of $S$ into $C(S)$ continuous, and thus for $\mu$ in $C(S)^{*}, \int f(x y) \mu(d x)$ is continuous in $y$. Consequently we can form the iterated integral $\iint f(x y) \mu(d x) \nu(d y)$ which, as a function of $f$, lies in $C(S)^{*}$. The corresponding measure $\mu \nu$, the convolution of $\mu$ and $\nu$, thus satisfies

$$
\int f(x) \mu_{\nu}(d x)=\iint f(x y) \mu(d x) \nu(d y)
$$

for $f$ in $C(S)$. Moreover using the monotoneity arguments of [6] we have (1.21) holding for bounded Baire functions $f$. Since the associative law is easily verified, and $\mu, \nu \geqq 0$ implies $\mu \nu \geqq 0$ while $\|\mu\|=\mu(1)=$ $\int \mu(d x)$ for $\mu \geqq 0, \tilde{S}$ clearly forms a semigroup under convolution, abelian if $S$ is (by Fubini's theorem); similarly $\widetilde{\tilde{S}}$ forms a semigroup since clearly $\|\mu \nu\| \leqq\|\mu\| \cdot\|\nu\|$. If we now add the $\omega^{*}$ topology we obtain compact semigroups: for since $y \rightarrow f^{y}$ is continuous for an $f$ in $C(S)$, $F=\left\{f^{y}: y \in S\right\}$ is a compact subset of $C(S)$, and thus pointwise convergence of an equicontinuous bounded net of functions on $F$ implies uniform convergence by Ascoli's theorem. But $\tilde{S}$ and $\tilde{\tilde{S}}$ are equicontinuous sets of functions on $F$ and $\omega^{*}$ convergence amounts to pointwise convergence, so $\mu_{\delta} \rightarrow \mu, \nu_{\delta} \rightarrow \nu$ imply $\int f(x y) \mu_{\delta}(d x) \rightarrow \int f(x y) \mu(d x)$ uniformly in $y$ and therefore

$$
\iint f(x y) \mu_{\delta}(d x) \nu_{\delta}(d y) \rightarrow \iint f(x y) \mu(d x) \nu(d y), \text { or } \mu_{\delta} \nu_{\delta} \rightarrow \mu_{\nu} .
$$

Finally we note the existence, for each non-negative regular Borel measure $\mu$, of a unique closed set $A=$ carrier $\mu \subset S$ with the property that $\mu A=\|\mu\|$ and $\mu U>0$ for each open $U$ with $A \cap U \neq \phi[10]$;

1 For $\mu, \vee \geqq 0$ one argues as follows: the set of non-negative Baire $f$ for which

$$
f^{\prime}(y)=\int f(x y) \wedge 1 \mu(d x)
$$

defines a Baire function $f^{\prime}$ and for which

$$
\iint f(x y) \wedge 1 \mu(d x)_{\vee}(d y)=\int f(x) \wedge 1 \mu \nu(d x)
$$

is clearly a monotone class containing the non-negative elements of $C^{R}(S)$, and thus includes all non-negative Baire $f$. For general $\mu, \nu$ the decomposition $\mu=\mu_{1}-\mu_{2}+i\left(\mu_{3}-\mu_{4}\right)$, and the obvious distributivity of convolution suffice. Also monotoneity shows $f$ Baire on $S$ implies $\tilde{f}:(x, y) \rightarrow f(x y)$ is a Baire function on $S \times S$, and thus Fubini's theorem may be applied to $\tilde{f}$. 
it is simply the complement of the union of all open sets of $\mu$ measure zero.

2. Idempotents and subgroups. The fundamental tool in our analysis is the following extension of Wendel's Lemma 4.

LEMMA 2.1 For $\mu$ and $\nu$ in $\widetilde{S}$,

$$
\text { carrier } \mu_{\nu}=(\text { carrier } \mu) \cdot(\text { carrier } \nu) \text {. }
$$

Proof. Let $A$ and $B$ be the respective carriers of $\mu$ and $\nu$. Since each is compact so is $A \cdot B$, which in particular is then a Borel set. Thus by the regularity of $\mu \nu$, for $\varepsilon>0$ we have an open $U$ containing $A \cdot B$ for which $\mu_{\nu}(U) \leqq \mu_{\nu}(A \cdot B)+\varepsilon$. Since $S$ is normal Urysohn's lemma applies to yield an $F$ in $C(S)$ with $\varphi_{A \cdot B} \leqq F \leqq \varphi_{U}$ (where $\mu_{E}$ is the characteristic function of $E$ ), i.e., $0 \leqq F \leqq 1$ and $F=0$ on $U^{\prime}$, =1 on $A \cdot B$. But it is clear that $\varphi_{A}(x) \varphi_{B}(y) \leqq F(x y)$ for all $x, y$ in $S$, and thus

$$
\begin{aligned}
1=\mu(A) \cdot \nu(B) & =\iint \varphi_{A}(x) \varphi_{B}(y) \mu(d x) \nu(d y) \leqq \iint F(x y) \mu(d x) \nu(d y) \\
& =\int F(x) \mu_{\nu}(d x) \leqq \mu_{\nu}(U) \leqq \mu_{\nu}(A \cdot B)+\varepsilon \leqq 1+\varepsilon .
\end{aligned}
$$

Since $\varepsilon>0$ is arbitrary, $\mu \nu(A \cdot B)=1$. Moreover if $U$ is now anopen set with $(A \cdot B) \cap U \neq \phi$ then we can find open sets $V$ and $W$ for which $V \cap A \neq \phi, W \cap B \neq \phi$, and $V^{-} \cdot W^{-} \subset U$; choosing an $F$ in $C(S)$ with $\varphi_{V^{-} \cdot W^{-}} \leqq F \leqq \varphi_{U}$ again yields $\mu\left(V^{-}\right) \cdot \nu\left(W^{-}\right) \leqq \mu_{\nu}(U)$, and this combines with $\mu\left(V^{-}\right) \geqq \mu(V)>0, \nu\left(W^{-}\right) \geqq \nu(W)>0$ to show $\mu \nu(U)>0$. Hence $A \cdot B$ is indeed the carrier of $\mu \nu$.

If $\mu$ is now an idempotent in $\widetilde{S}$,

$$
(\text { carrier } \mu)^{2}=\text { carrier } \mu^{2}=\text { carrier } \mu \text {. }
$$

In the group situation this guarantees the carrier is a group [4, 7], but in the case of an abelian semigroup $S$ we must go further.

THEOREM 2.2 Let $S$ be abelian or a group, and $\mu^{2}=\mu \in \tilde{S}$. Then carrier $\mu$ is a subgroup of $S$ and $\mu$ is its Haar measure".

Proof (Following Wendel). For completeness we shall include both cases in our proof, although in the group situation we have Wendel's Theorem 1. Let $H=$ carrier $\mu$, so $H^{2}=H$. For $f$ in $C^{R}(S)$ (the space

2 For non-abelian $S$ this and our subsequent results fail in general. For take $S=$ $[0,1]$ under $\odot$, where $x \circ y=y$. Then $\mu \nu=\nu$, for $\mu, \nu \approx \tilde{S}$ and each element of $\tilde{S}$ is an idempotent. 
of real valued elements of $C(S))$ let $f^{\prime}(x)=\int f(y x) \mu(d y), x \in S$, so that $f^{\prime} \in C^{R}(S)$. Since $H$ is compact $f^{\prime}$ assumes its supremum over $H$ at some $x_{0}$ in $H$, and

$$
\begin{aligned}
f^{\prime}\left(x_{0}\right)=\int f^{x_{0}}(y) \mu(d y)= & \mu\left(f^{x_{0}}\right)=\mu^{2}\left(f^{x_{0}}\right) \\
& =\iint f\left(y z x_{0}\right) \mu(d y) \mu(d z)=\int f^{\prime}\left(z x_{0}\right) \mu(d z) \leqq f^{\prime}\left(x_{0}\right)
\end{aligned}
$$

since $f^{\prime} \leqq f^{\prime}\left(x_{0}\right)$ on (carrier $\left.\mu\right) \cdot x_{0}=H x_{0} \subset H^{2}=H$. Consequently $f^{\prime}\left(x_{0}\right)$ $=\int f^{\prime}\left(z x_{0}\right) \mu(d z)$ and, since $f^{\prime}$ is continuous and $H=$ carrier $\mu, f^{\prime}$ assumes its supremum over $H$ on all of $H x_{0}$; in particular then on the least ideal $I$ of the subsemigroup $H$ of $S$.

(In case $S$ is a group our proof is complete: for $H$ is a group, $I=H$, and $f^{\prime}$ constant on $I=H \Rightarrow \mu$ is right invariant).

Now suppose $H \backslash I \neq \phi$. Then we can find an $x_{1}$ in $H$ and nonnegative $f$ in $C(S)$ vanishing on $I$ for which $f^{x_{1}}$ does not vanish on all of $H$; otherwise for each $f \geqq 0$ in $C(S)$ with $f(I)=0$ we have $f^{x}(H)$ $=f(H x)=0$ for all $x$ in $H$, and thus $f(H)=f\left(H^{2}\right)=0$. Hence for this $f$ and $x_{1}$ we have $\mu\left(f^{x_{1}}\right)>0$ while, for $y$ in $I, f^{\prime}(y)=\int f(x y) \mu(d x)=$ $\int 0 \mu(d x)=0$ since $H y \subset I$ and $f$ vanishes on $I$. But since $f^{\prime}$ assumes its supremum over $H$ on $I, 0 \geqq f^{\prime}\left(x_{1}\right)=\mu\left(f^{x_{1}}\right)>0$, the desired contradiction, and $H=I$, a subgroup (of $H$ and thus) of $S$. Moreover since $f^{\prime}$ is constant on $I=H, \mu$ is invariant and our proof complete.

For a subset $E$ of $\tilde{S}$ we shall refer to $\left(\bigcup_{\mu \in E} \text { carrier } \mu\right)^{-}$as the carrier of $E$, which is obviously consistent with our former use of the term. It should be noted that if $E$ is a subsemigroup of $\widetilde{S}$ then carrier $E$ is a subsemigroup of $S$. For by Lemma $2.1 \bigcup_{\mu \in E}$ carrier $\mu$ is closed under multiplication, and therefore its closure is also. Moreover carrier $E^{-}=$ carrier $E$; for if carrier $\mu \not \subset$ carrier $E$ then there is an $f$ in $C(S)$ vanishing on carrier $E$ with $\mu(f) \neq 0$. But then $\nu(f)=0$ for $\nu$ in $E$ and therefore for $\nu$ in $E^{-}$as well, and $\mu \notin E^{-}$.

Theorem 2.3 Let $S$ be either abelian or a group, and let $\Gamma$ be a subgroup of $\tilde{S}$. Then the carrier $G$ of $\Gamma$ is a subgroup of $S$ while the carrier $g$ of the identity $\eta$ of $\Gamma$ is a normal subgroup of $G$. If $T_{\eta}$ denotes the map of $(G / g)^{-} \rightarrow \widetilde{S}$ defined by

$$
T_{\eta} \nu(f)=\int_{\alpha / g} \int_{g} f(x y) \eta(d x) \nu(d y g), \quad f \in C(S),
$$

then $T_{\eta}$ takes $(G / g)^{\sim}$ onto the $\omega^{*}$ closed convex hull $\mathscr{C}\left(I^{\prime}\right)$ of $\Gamma$, the point masses $(G / g)^{p}$ of $G / g$ onto $\Gamma$, and in each case is a (topological) isomorphism 
between these semigroups.

CoRollary 2.31. $I$ is the set of $G$-translates of Haar measure on $g$. For $\eta$ is Haar measure on $g$ by Theorem 2.2 and thus for $\nu=$ mass 1 at $g y \in G / g$ we have $T_{\eta} \nu(f)=\int_{g} f(x y) r(d x)$, which of course corresponds to the Haar measure of $g$ translated to the coset $g y$.

Proof of Theorem 2.3. Consider first the case in which $S$ is abelian. Let $S_{0}=\mathbf{U}_{\mu \in \Gamma}$ carrier $\mu$, an algebraic subsemigroup of $S$ with $S_{0}^{-}=$ carrier $\Gamma=G$. Since $\mu=\eta \mu$ for $\mu$ in $\Gamma$, carrier $\mu=$ carrier $\eta \cdot$ carrier $\mu$ $=g$ carrier $\mu$ by Lemma 2.1, and thus $g S_{0}=S_{0}$ and therefore $g G=G$.

But for $x$ in $S_{0}$ we have $x \in$ carrier $\mu, \mu \in \Gamma$, so $x \cdot$ carrier $\mu^{-1} \subset$ carrier $\mu \mu^{-1}=g$ and $x G \cap g \neq \phi$. Further, since $x y G \subset x G \cap y G$ for $x, y \in S_{0} \subset G$ we conclude from the compactness of $g$ that $g$ meets $\bigcap_{x \in S_{0}} x G$, the least ideal $I$ of the compact semigroup $G$ (cf. (1.11)). Consequently $g \subset I$; for $i \in g \cap I$ implies $g=i g \subset I$ since $g$ is a group and $I$ an ideal. Since $g G=G$ we obtain $G \subset I \subset G$, and since $I$ is algebraically a group, $G$ is a subgroup of $S$.

Now evidently $T_{\eta}$ maps $(G / g)^{\sim}$ into $\tilde{S}$. Let $f$ be in $C(S)$ and vanish on $G$. Then clearly $T_{\eta} \nu(f)=0, \nu \in(G / g)^{2}$, so that $G$ contains the carrier of any measure in the range of $T_{\eta}$. The subset $M$ of $\tilde{S}$ of elements with carriers contained in $G$ may be considered as a subset of either $C(S)^{*}$ or $C(G)^{*}$; in each case we obtain the same $\omega^{*}$ topology since by Urysohn's lemma $C(G)$ is exactly the set of restrictions to $G$ of the elements of $C(S)$, and $\mu(f)=\mu(f \mid G)$ (where $\mu$ on the left is in $C(S)^{*}$, and on the right in $\left.C(G)^{*}\right)$. For the same reason we may evidently form the convolution of two elements of $M$ in either place, i.e. $M$ may be considered as a subsemigroup of either $\tilde{S}$ or $\tilde{G}$. Thus it will clearly suffice to consider $T_{\eta}$ as a map of $(G / g)^{-}$into $\tilde{G}$.

But now we recognize $T_{\eta}$ as (a restriction of) the adjoint of the map $f \rightarrow f^{\prime}$ of $C(G) \rightarrow C(G / g)$ defined by setting $f^{\prime}(y g)=\int_{g} f(x y) \eta(d x)$. Thus $T_{\eta}$ is $\left(\omega^{*} \rightarrow \omega^{*}\right)$ continuous, and since $f \rightarrow f^{\prime}$ is onto $[6,9], T_{\eta}$ is one-toone, hence a homeomorphism on $(G / g)^{2}$. Further $T_{\eta}$ is an isomorphism since for $f \in C(G)$

$$
\begin{aligned}
T_{\eta \nu_{1}} \cdot T_{\eta} \nu_{2}(f) & =\int_{G / g} \int_{g} \int_{G / g} \int_{g} f(x y z w) \eta(d x) \nu_{1}(d y g) \eta(d z) \nu_{2}(d w g), \\
T_{\eta}\left(\nu_{1} \nu_{2}\right)(f) & =\int_{G / g} \int_{g} f(x y) \eta(d x) \nu_{1} \nu_{\nu_{2}}(d y g) \\
& =\int_{G / g} \int_{G / g} \int_{g} f(x y w) \eta(d x) \nu_{1}(d y g) \nu_{\nu_{2}}(d w g) \\
& =\int_{G / g} \int_{G / g} \int_{g} \int_{g} f(x z y w) \eta(d x) \eta(d z) \nu_{1}(d y g) \nu_{2}(d w g)
\end{aligned}
$$


since $\eta^{2}=\eta$, and thus multiplicativity follows from Fubini's theorem and commutativity.

Now let $\rho$ be the canonical homomorphism of $G \rightarrow G / g$ and, for $\mu$ in $\Gamma$ define $\bar{\mu} \in(G / g)^{2}$ by $\bar{\mu}(F)=\mu(F \circ \rho), F \in C(G / g)$. Then for $f \in C(G)$,

$$
\begin{aligned}
\mu(f)=\eta \mu(f)=\int_{G} \int_{g} f(x y) \eta(d x) \mu(d y)=\int_{G / g} \int_{g} f(x y) \eta(d x) \bar{\mu}(d y g) & \\
& =T_{\eta} \bar{\mu}(f), \quad \text { so } \quad \Gamma \subset T_{\eta}(G / g)^{2} .
\end{aligned}
$$

Thus the (compact) preimage of $\Gamma$ is a subgroup of $(G / g)^{2}$ whose identity is the mass 1 at the identity $g$ of $G / g$ (for clearly this measure maps onto $\eta$ and $T_{\eta}$ is one-to-one). Since $G / g$ is a group, Lemma 2.1 implies each element of the preimage is a point mass; indeed the preimage consists of just those obtained from a closed subgroup of $G / g$ since as is well known the map from points to point masses (in the $\omega^{*}$ topology) is a homeomorphism [2] and trivially a group isomorphism. Hence we may identify the preimage as $\left(G_{0} / g\right)^{p}$, the point masses on $G_{0} / g$ where $G_{0}$ is a subgroup of $G$ containing $g\left(G_{0}\right.$ is closed since $G_{0} / g$ and $g$ compact imply $G_{0}$ is compact). But obviously the carrier of each element of $T_{\eta}\left(G_{0} / g\right)^{p}$ is contained in $G_{0}$ so that carrier $\Gamma=G \subset G_{0}$, and $G_{0}=G$, $T_{\eta}(G / g)^{p}=\Gamma$.

To complete the proof in the abelian case we need only note the well known fact [2], that $(G / g)^{\sim}$ is the $\omega^{*}$ closed convex hull of $(G / g)^{p}$, so that $T_{\eta}\left[(G / g)^{\sim}\right]=\mathscr{C}(\Gamma)$ follows from linearity and continuity.

Now suppose $S$ is a (non-abelian) compact group with identity $e$. Since we clearly have $G=$ carrier $\Gamma=G^{2}, G$ is a subgroup of $S[4,7]$. Moreover $g$ is a normal subgroup of $G$. For $x \in$ carrier $\mu, \mu \in \Gamma$, implies $x$ carrier $\mu^{-1} \subset g$ by Lemma 2.1 so that if $y \in$ carrier $\mu^{-1}$,

$$
x y=z \in g, x^{-1}=y z^{-1} \in\left(\text { carrier } \mu^{-1}\right) \cdot g=\text { carrier } \mu^{-1} .
$$

Thus

$$
x^{-1} g x \subset \text { carrier } \mu^{-1} \cdot g \cdot \operatorname{carrier} \mu=g,
$$

and $x^{-1} g x \subset g$ for a dense set of $x$ in $G$; if $y \in g$ then $x^{-1} y x \in g$ for all $x$ in $G$, by continuity, and $g$ is normal in $G$.

Now if we omit the first two paragraphs of the proof for the abelian case, each step will apply here with one exception: the proof that $T_{\eta}$ is multiplicative. But (applying Fubini's theorem) this follows from the fact that

$$
\int_{g} f(x y z w) \eta(d z)=\int_{g} f(x z y w) \eta(d z)
$$

or equivalently

$$
\int_{g} f_{x}^{\omega}(y z) \eta(d z)=\int_{g} f_{x}^{\omega}(z y) \eta(d z)
$$

and thus ultimately from $y g=g y$. 
2.4 Remark. If $\Gamma$ is an algebraic subgroup of $\tilde{S}$ then $\Gamma^{-}$is a subgroup of $\tilde{S}$ so that $I^{\prime}$ consists of the $G$-translates of a Haar measure, where $G$ is an algebraic subgroup of $S$. For if the net $\left\{\mu_{\delta}\right\} \subset \Gamma$ converges to $\mu \in \Gamma^{-}$, then any cluster point $\nu$ of $\left\{\mu_{\delta}^{-1}\right\}$ must satisfy $\mu_{\nu}=\eta$ as a cluster point of $\left\{\mu_{\delta} \mu_{\delta}^{-1}\right\}$; and clearly $\mu=\mu \eta$.

3. Least ideals and carriers. Our next result gives the relationship when $S$ is abelian, between the least ideal of a subsemigroup of $\widetilde{S}$ and the least ideal of its carrier: the carrier of the least ideal is the least ideal of the carrier.

THEOREM 3.1. Let $S$ be abelian and let $\Sigma$ be a subsemigroup of $\tilde{S}$ with least ideal $\mathscr{I}$; let $S_{1}=$ carrier $\Sigma$ with least ideal $I$. Then $I=$ carrier $\mathscr{I}$.

Proof. We know that $\mathscr{F}$ is a subgroup of $\Sigma$ and thus of $\widetilde{S}$. Hence by Theorem 2.3 its carrier is a subgroup $G$ of $S$. Let $S_{0}=\bigcup_{\mu \in \Sigma}$ carrier $\mu$, a dense algebraic subsemigroup of $S_{1}$. Let $x \in S_{0}$ so that $x \in$ carrier $\mu$ for some $\mu$ in $\Sigma$. For $\nu$ in $\mathscr{J}, \mu \nu \in \mathscr{J}$ so $x$ carrier $\nu \subset$ carrier $\mu \nu \subset G$ by Lemma 2.1, and thus $x S_{0} \cap G \neq \phi$ and $x S_{1} \cap G \neq \phi$. Since $I=$ $\bigcap_{x \in S_{0}} x S_{1}$ by (1.11) we conclude as in the proof of 2.3 that $G \cap I \neq \phi$ and therefore $G \subset I$.

But the fact that $x$ carrier $\nu \subset G$ for $x \in S_{0}, \nu \in \mathscr{S}$ clearly implies $x G \subset G$ for $x \in S_{0}$. Consequently for $y$ in $G, x y \in G$ for all $x$ in $S_{1}$, by continuity, and thus $S_{1} G \subset G$, or $G$ is an ideal in $S_{1}$. Hence $G$ contains the least ideal $I$ and $I=G=$ carrier $\mathscr{I}$.

Theorem 3.2. Let $\mu \in \widetilde{S}$, with $S$ abelian. Then $N^{-1} \sum_{n=1}^{N} \mu^{n} \rightarrow$ Haar measure on the least ideal of the subsemigroup of $S$ generated by carrier $\mu$. If $S$ is $a$ (not necessarily abelian) group, $N^{-1} \sum_{n=1}^{N} \mu^{n} \rightarrow$ Haar measure of the subgroup of $S$ generated by carrier $\mu$.

Proof. Let $\Sigma_{\mu}$ be the subsemigroup of $\tilde{S}$ generated by $\mu, \nu_{N}=$ $N^{-1} \sum_{n=}^{N} \mu^{n}$, and let $\nu$ be any cluster point of $\left\{\nu_{N}\right\}$ which of course must lie in $\mathscr{C}\left(\Sigma_{\mu}\right)$. Since $\left\|\mu_{\nu}-\nu_{N}\right\| \rightarrow 0$ we have $\mu_{\nu}=\nu$ and thus $\lambda_{\nu}=\nu$ for each $\lambda \in \mathscr{C}\left(\Sigma_{\mu}\right)$. Since $\mathscr{C}\left(\Sigma_{\mu}\right)$ is abelian this clearly implies $\nu$ is the unique cluster point of $\left\{\nu_{N}\right\}$ so that $\nu_{N} \rightarrow \nu$ by compactness. Moreover $\lambda \nu=\nu, \lambda \in \mathscr{C}\left(\Sigma_{\mu}\right)$, says $\{\nu\}$ is the least ideal of the subsemigroup $\mathscr{C}\left(\Sigma_{\mu}\right)$ of $\widetilde{S}$, and an idempotent, so that $\nu$ is Haar measure of its carrier by 2.2 .

Now if $S$ is abelian the carrier of $\nu$ is the least ideal $I$ of carrier $\mathscr{C}\left(\Sigma_{\mu}\right)$ by 3.1. Evidently the carrier of the algebraic convex hull of $\Sigma_{\mu}$ coincides with the carrier of $\Sigma_{\mu}$, and since carrier $E^{-}=$carrier $E$, we have carrier $\mathscr{C}\left(\Sigma_{\mu}\right)=$ carrier $\Sigma_{\mu}$ and our proof is complete in this case.

If $S$ is a group with identity $e$, let $G$ be the subgroup of $S$ generated 
by carrier $\mu$. Since a subsemigroup of a compact group is a group, carrier $\left\{\mu^{n}: n \geqq 1\right\}$ is a subgroup of $S$ and clearly must coincide with $G$. Thus if $f(G)=0, f \in C(S)$ we have $f$ vanishing on carrier $\mu^{n}$, hence $\mu^{n}(f)=0$, all $n$, and $\nu(f)=0$; consequently carrier $\nu \subset G$. On the other hand $\mu^{n} \nu=\nu$ so that by 2.1 carrier $\mu^{n} \cdot$ carrier $\nu=$ carrier $\nu$ and thus

$\bigcup$ carrier $\mu^{n}=\mathbf{U}\left(\right.$ carrier $\left.\mu^{n} e\right) \subset \bigcup \mathbf{U}\left(\right.$ carrier $\left.\mu^{n} \cdot \operatorname{carrier} \nu\right)=$ carrier $\nu$ so $G \subset$ carrier $\nu$ and our proof is complete.

3.3 Remark. More generally we can follow Alaoglu and Birkhoff [1] to obtain a stronger assertion. Let $E$ be a commuting subset of $\tilde{S \text {, and }}$ let $\Sigma$ be the abelian subsemigroup of $\tilde{S}$ generated by $E$. We can regard $\mathscr{C}(\Sigma)$ as partially ordered by $\mu \leqq \nu \Leftrightarrow \nu \in \mu \Sigma$, and then $\mathscr{C}(\Sigma)$ forms a directed set $(\mu \nu \geqq \mu, \nu)$. If we regard $\mathscr{C}(\Sigma)$ as indexed by itself then $\mathscr{C}(\Sigma)$ is a net and the net converges to Haar measure on the least ideal of carrier $\Sigma$. For given $\mu \in \mathscr{C}(\Sigma)$ and $\varepsilon>0$ there is a $\nu_{0}$ in $\mathscr{C}(\Sigma)$ for which $\left\|\mu_{\nu}-\nu\right\|<\varepsilon, \nu \geqq \nu_{0}$ : simply choose $\nu_{0}=N^{-1} \sum_{n=1}^{N} \mu^{n}$ for $N$ large enough to yield $\left\|\mu_{0}-\nu_{0}\right\|<\varepsilon$; then $\nu \geqq \nu_{0} \Rightarrow \nu=\nu_{0} \lambda$, $\|\mu \nu-\nu\|=$ $\left\|\mu \nu_{0} \lambda-\nu_{0} \lambda\right\| \leqq\left\|\mu \nu_{0}-\nu_{0}\right\| \cdot\|\lambda\|<\varepsilon$. Consequently we obtain a unique cluster point $\nu$ of our net to which the net must converge, with $\mu_{\nu}=\nu$, $\mu \in \mathscr{C}(\Sigma)$ and the remainder of our proof applies.

3.4. Our next result gives more explicit information about the least ideal of a subsemigroup of $\tilde{S}$ when $S$ is abelian.

THEOREM 3.5. Let $S$ be abelian, and $\Sigma$ be the subsemigroup of $\tilde{S}$ generated by a subset $E$ of $\widetilde{S}$, with carrier $S_{1}$. Let $\mathscr{I}$ and $I$ be the respective least ideals of $\Sigma$ and $S_{1}$ with identities $\eta$ and $e$ respectively. Then $\mathscr{F}$ is the set of I-translates of Haar measure $\eta$ of the subgroup $h$ of I generated by $\left\{(e\right.$ carrier $\left.\mu)(e \text { carrier } \mu)^{-1}: \mu \in E\right\}$.

Proof. We already know from Theorem 3.1 and Corollary 2.31 that $\mathscr{F}$ is the set of $I$-translates of Haar measure $\eta$ of some subgroup $g$ of $I$; we have only to show $g=h$. But each subgroup $g_{0}$ of $I$ is determined by its orthogonal subgroup $g_{0}^{\perp}=\left\{\alpha \in \hat{I}: \alpha\left(g_{0}\right)=1\right\}$ in the character group $\hat{I}$ of $I$, so we need only show $g^{\perp}=h^{\perp}$. Moreover the elements of $g^{\perp}$ are just those $\alpha$ in $\hat{I}$ for which $\eta(\alpha)=\int \alpha(x) \eta(d x)=1$ (for all others $\eta(\alpha)=0$ ), hence $g^{\perp}=\{\alpha \in \hat{I}:|\mu(\alpha)|=1, \mu \in \mathscr{I}\}$.

Now for $\mu$ in $\Sigma, e$ carrier $\mu \subset$ carrier $\eta$ carrier $\mu=$ carrier $\eta \mu \subset I$ and since $\eta \mu \in \mathscr{F}$, carrier $\eta \mu$ is a coset $y g \subset I$. Thus

$$
(e \text { carrier } \mu)(e \text { carrier } \mu)^{-1} \subset y g(y g)^{-1}=g \text {, }
$$

and $\alpha \in g^{\perp}$ implies $\alpha \in h^{\perp}$. To see that $h^{\perp} \subset g^{+}$, note that each $\alpha$ 
in $\hat{I}$ has a continuous multiplicative extension $\alpha^{*}$ to $S_{1}$ : simply set $\alpha^{*}(x)=\alpha(x e), x \in S_{1}$. Further $\alpha^{*}$ has a continuous extension $\alpha^{\prime}$ to all of $S$ by Urysohn's lemma, and, for $\mu$ in $\Sigma, \mu\left(\alpha^{\prime}\right)=\mu\left(\alpha^{*}\right)$; thus if $\mu_{\delta} \rightarrow \mu$ in $\Sigma, \mu_{\delta}\left(\alpha^{*}\right) \rightarrow \mu\left(\alpha^{*}\right)$. But since $\alpha^{*}$ is multiplicative if we define the (Fourier) transform $\hat{\mu} \in C(\hat{I})$ of $\mu$ in $\Sigma$ by setting $\hat{\mu}(\alpha)=\mu\left(\alpha^{*}\right)=$ $\int \alpha^{*}(x) \mu(d x)$, we have $\left(\mu_{\nu}\right)^{\wedge}=\hat{\mu} \cdot \hat{\nu}$ (ordinary product in $\left.C(\hat{I})\right)$, with $\hat{\mu}(\alpha)$ $=\mu(\alpha)$ for $\mu$ in $\mathscr{F}$ (since carrier $\mu$ is then $\subset I$ ).

Let $\alpha \in h^{\perp}$. Then

$\alpha\left((e\right.$ carrier $\left.\mu)(e \text { carrier } \mu)^{-1}\right)=\alpha(e$ carrier $\mu) \cdot \overline{\alpha(e \text { carrier } \mu)}=1$

for $\mu$ in $E$ which implies $\alpha$ is constant on the sets $e$ carrier $\mu, \mu$ in $E$. Thus

$$
\hat{\mu}(\alpha)=\int \alpha^{*}(x) \mu(d x)=\int \alpha(x e) \mu(d x)
$$

is a unimodular complex number. But then $(\mu \nu)^{\wedge}=\hat{\mu} \cdot \hat{\nu}$ implies that $|\hat{\mu}(\alpha)|=1$ for $\mu$ in the algebraic subsemigroup of $S$ generated by $E$; since $\mu_{\delta} \rightarrow \mu$ in $\Sigma$ implies $\hat{\mu}_{\delta}(\alpha)=\mu_{\delta}\left(\alpha^{*}\right) \rightarrow \mu\left(\alpha^{*}\right)=\hat{\mu}(\alpha)$ the same must be true for all $\mu$ in $\Sigma$. In particular for $\mu$ in $\mathscr{J},|\mu(\alpha)|=|\hat{\mu}(\alpha)|=1$ whence $\alpha \in g^{\perp}$ and $g^{\perp}=h^{\perp}$.

4. The semigroup $\tilde{\tilde{S}}$. When $S$ is abelian the subgroups of $\tilde{\tilde{S}}$, the convolution semigroup formed by the unit ball of $C(S)^{*}$, can be determined from those of $\widehat{S}$.

Let $\Gamma$ be a non-trivial (i.e. $\neq\{0\}$ ) subgroup of $\tilde{\widetilde{S}}$, with identity $\eta$. Clearly $0 \notin \Gamma$ and consequently $\|\mu\|=1$ for $\mu$ in $\Gamma$; for $\|\mu\|<1$ implies $\mu^{n} \rightarrow 0$ and thus $0 \in \Gamma$. Now by the Radon-Nikodym theorem we can associate with each complex measure $\mu$ a non-negative measure $|\mu|$ and a unimodular Baire function $\rho_{\mu}$ for which $\mu(d x)=\rho_{\mu}(x)|\mu|(d x)$ (we shall express this by writing $\mu=\rho_{\mu} \cdot|\mu|$ ) and $\||\mu|\|=\|\mu\|$. For write $\mu=\mu_{1}-\mu_{3}+i\left(\mu_{3}-\mu_{4}\right)$ with $\mu_{j} \geqq 0$, and let $\nu=\mu_{1}+\mu_{2}+\mu_{3}+\mu_{4}$. Each $\mu_{j}$ is absolutely continuous with respect to $\nu$ so there are functions $f_{j}$ in $L_{1}(\nu)$ (which we can take to be Baire functions since each $\nu$ integrable function is equivalent to a Baire function) for which $\mu_{j}=f_{j} \cdot \nu$. Set

$$
f=f_{1}-f_{2}+i\left(f_{3}-f_{4}\right), \quad|\mu|(d x)=|f(x)| \nu(d x)
$$

and $\rho_{\mu}(x)=f(x) /|f(x)|$ unless $f(x)=0$ when we set $\rho_{\mu}(x)=1$. Clearly $\rho_{\mu}$ and $|\mu|$ have the required properties.

Thus for $\mu$ and $\nu$ in $I$ we have

$$
\begin{array}{r}
1=\left\|\left|\mu_{\nu}\right|\right\|=\int 1\left|\mu_{\nu}\right|(d x)=\int \frac{1}{\rho_{\mu \nu}(x)} \mu \nu(d x)=\iint_{\rho_{\mu \nu}(x y)} \frac{1}{(d x) \nu(d y)} \\
=\iint \frac{\rho_{\mu}(x) \rho_{\nu}(y)}{\rho_{\mu \nu}(x y)}|\mu|(d x)|\nu|(d y) \leqq \iint 1|\mu|(d x)|\nu|(d y)=1 .
\end{array}
$$


Consequently we have a $|\nu|$ null set $E$ for which $y \notin E$ implies there is a $|\mu|$ null set $E_{y}$ for which $x \notin E_{y}$ implies $\rho_{\mu}(x) \rho_{\nu}(y)=\rho_{\mu \nu}(x y)$. Hence for $f$ in $C(S)$

$$
\begin{aligned}
\left|\mu_{\nu}\right|(f)=\int f(x) \frac{1}{\rho_{\mu \nu}(x)} \mu_{\nu}(d x) & =\iint f(x y) \frac{1}{\rho_{\mu \nu}(x y)} \mu(d x) \nu(d y) \\
= & \iint f(x y) \frac{\rho_{\mu}(x) \rho_{\nu}(y)}{\rho_{\mu \nu}(x y)}|\mu|(d x)|\nu|(d y) \\
& =\iint f(x y)|\mu|(d x)|\nu|(d y)=|\mu \| \nu|(f),
\end{aligned}
$$

so that $\mu \rightarrow|\mu|$ is an (algebraic) homomorphism of $\Gamma$ onto an algebraic subgroup $\Gamma_{0}$ of $\widetilde{S}$, whose identity is obviously $|\eta|$. Let $G=\operatorname{carrier} \Gamma_{0}$ $=$ carrier $\Gamma_{0}^{-}$, so that $\Gamma_{0}^{-}$consists of all $G$-translates of Haar measure $|\eta|$ of a subgroup $g$ of $G$. We shall see later that $\mu \rightarrow|\mu|$ is also continuous, so that $\Gamma_{0}$ is compact and coincides with $\Gamma_{0}^{-}$.

Now each Baire function $f$ on $S$ has its restriction to $g$ a Baire function of $g$ (for the set of real valued $f$ 's for which this holds is a monotone class containing $C^{R}(S)$ ). Thus $\rho_{\eta} / g$ is a Baire function on $g$. Applying (4.1) to the special case $\mu=\nu=\eta$ we conclude that there is an $|\eta|$ null set $E$ of $g$ for which $y \notin E$ implies there is an $|\eta|$ null set $E_{y}$ of $g$ for which $x \notin E_{y}$ implies $\rho_{\eta}(x) \rho_{\eta}(y)=\rho_{\eta}(x y)$. For simplicity let us now write $\rho_{\eta}=\rho$, and, restricting our attention entirely to $g$, write $d x$ for $|\eta|(d x)$, the element of Haar measure on $g$, and speak of $|\eta|$ null sets as null.

For $f \in L_{1}(g)$ (which we take as a Baire function of $g$ ) let $M(f)=$ $\int f(x) \rho(x) d x$. Since $y \cdot E_{y}$ is null by translation invariance, and $x \notin y E_{y}$ implies $y^{-1} x \notin E_{y}$ and thus $\rho\left(y^{-1} x\right) \rho(y)=\rho\left(y^{-1} x y\right)=\rho(x)$ for $y \notin E$, we can write (with $f * h$ the usual convolution in $L_{1}(g)$ )

$$
\begin{aligned}
& M(f * h)= \int f * h(x) \rho(x) d x=\iint f(y) h\left(y^{-1} x\right) \rho(x) d y d x \\
&=\iint f(y) h\left(y^{-1} x\right) \rho(x) d x d y=\iint f(y) h\left(y^{-1} x\right) \rho\left(y^{-1} x\right) \rho(y) d x d y \\
& \quad=\int f(y) M(h) \rho(y) d y=M(f) \cdot M(h)
\end{aligned}
$$

so that $M$ is a multiplicative functional on $L_{1}(g)$. Thus we have a character $\beta$ of $g$ for which $\rho=\beta \bmod |\eta|$ on the carrier $g$ of $|\eta|$, and clearly then $\eta(d x)=\beta(x)|\eta|(d x)$. Moreover since $\hat{g}=\hat{G} / g^{\perp}$ we have an $\alpha$ in $\hat{G}$ for which $\alpha \mid g=\beta$, so that $\eta(d x)=\alpha(x)|\eta|(d x)$ as elements of $\tilde{S}$, or $\eta=\alpha \cdot|\eta|$. (Note that essential use is made here of the abelian nature of $S$ ).

Now $\alpha^{-1}$ can be extended continuously to all of $S$; since each element 
of $\Gamma$ vanishes on subsets of $G^{\prime}$ the map $\mu \rightarrow \alpha^{-1} \cdot \mu$ is clearly a one-toone continuous map on $\Gamma$, and therefore a homeomorphism. Further it is clearly multiplicative, so $\mu \rightarrow \alpha^{-1} \cdot \mu$ is an isomorphism of $\Gamma$ with a subgroup $\Gamma_{1}$ of $\tilde{\widetilde{S}}$. The identity of $\Gamma_{1}$ is $|\eta|$, and thus for $\nu$ in $\Gamma_{1}$,

$$
\iint \nu(d x) \nu^{-1}(d y)=\int|\eta|(d x)=1
$$

whence

$$
1 \leqq \int\left|\int \nu(d x)\right|\left|\nu^{-1}\right|(d y)=|\nu(1)| \cdot\left\|\nu^{-1}\right\| \leqq 1 .
$$

Consequently $\nu(1)$ is a unimodular complex number $\beta(\nu)$, and since $\nu_{1} \nu_{2}(1)=\nu_{1}(1) \nu_{2}(1), \beta$ is multiplicative ; evidently $\beta$ is continuous and thus a character of $\Gamma_{1}$. Moreover $\int(1 / \beta(\nu)) \nu(d x)=1$ implies $(1 / \beta(\nu)) \nu \geqq 0$, so the map $\tau: \nu \rightarrow \beta(\nu)^{-1} \nu$ is a continuous homomorphism of $\Gamma_{1}$ into a subgroup of $\tilde{S}$. Further the composition of $\tau$ with $\mu \rightarrow \alpha^{-1} \cdot \mu$, taking

$$
\mu \rightarrow \alpha^{-1} \cdot \mu \rightarrow \beta\left(\alpha^{-1} \cdot \mu\right)^{-1} \alpha^{-1} \cdot \mu=\lambda
$$

clearly must map $\mu \rightarrow|\mu|$ since $\mu=\beta\left(\alpha^{-1} \mu\right) \alpha \cdot \lambda$ with $\lambda \geqq 0$ and $\beta\left(\alpha^{-1} \cdot \mu\right) \alpha$ unimodular on $G$. Thus our original map $\mu \rightarrow|\mu|$ was continuous and $\tau: \nu \rightarrow \beta(\nu)^{-1} \nu$ maps $\Gamma_{1}$ onto $\Gamma_{0}$, which now appears as the full set of $G$-translates of Haar measure $|\eta|$ on $g$.

For $\nu$ in $\Gamma_{0}$ let $\tau^{-1} \nu=\Theta_{\nu} \cdot \nu$ where $\Theta_{\nu}$ is a closed subset of the circle group $T$, and in particular $\Theta=\Theta_{|\eta|}$ is a subgroup of $T$. Since $\Gamma_{1}=$ $\bigcup_{\nu \in \Gamma_{0}} \Theta_{\nu} \cdot \nu$ it remains to find the $\Theta_{\nu}$. But $\nu \in \Gamma_{0}, t \in T$ and $t \nu \in \Gamma_{1}$, imply $\beta(t \nu)=t$ since $\nu \geqq 0$ and $\beta\left(t_{\nu}\right)^{-1} t_{\nu} \geqq 0$; thus $\beta\left(\Theta_{\nu} \cdot \nu\right)=\Theta_{\nu}$. Moreover since $\nu \rightarrow \Theta_{\nu} \cdot \nu$ maps $\Gamma_{0}$ (topologically and) isomorphically onto $\Gamma_{1}|\Theta| \eta \mid$ and $\beta$ (taking $\Theta|\eta|$ onto $\Theta$ ) maps the quotient group $\Gamma_{1}|\Theta| \eta \mid$ of cosets into $T / \Theta$ in a homomorphic fashion, and continuously (as is easily seen), the composition $\nu \rightarrow \beta\left(\Theta_{\nu} \cdot \nu\right)=\Theta_{\nu} \in T / \Theta$ is continuous. We now distinguish two cases: $\Theta=T$ so that, as we could have seen earlier, $\Gamma_{1}=T \Gamma_{0}$ (clearly this occurs iff $\Gamma$ is circular in the sense that $T \Gamma \subset \Gamma$ ), or $\Theta$ is the group of $n$th roots of unity, $n \geqq 1$. In the latter case we may apply the natural isomorphism $\sigma\left(: \xi \rightarrow \xi^{n}\right)$ of $T / \Theta$ onto $T$ to map $\Theta_{\nu}$ into $T$. Writing $|\eta|_{x}\left(\varepsilon \Gamma_{0}\right)$ for the translate to $x g$ of $|\eta|$ we thus have $\varphi(x)=\sigma\left(\Theta_{|\eta|_{x}}\right)$ defining a character of $G$ lying in $g^{\perp}$ : for the map $x \rightarrow|\eta|_{x}$ of $G$ into $\Gamma_{0}$ is a continuous homomorphism as the composition of $G \rightarrow G / g$, the map from $G / g$ into $(G / g)^{p}(\subset \tilde{G})$ (continuous by [2]) followed by $T_{|\eta|}$ (cf. 2.3). Consequently $\Theta_{|\eta|_{x}}$ consists of just the $n$th roots of $\varphi(x)$ and we may express our general element of $\Gamma_{1}$ as $\varphi(x)^{1 / n}|\eta|_{x}$, where $x \in G$ and $\varphi(x)^{1 / n}$ denotes any root. In summary then, we have

THEOREM 4.2. Let $S$ be abelian and $\Gamma$ any non-trivial subgroup of 
$\tilde{\tilde{S}}$. If $\Gamma$ is circular in the sense that $T \Gamma \subset \Gamma$ then there are subgroups $g$ and $G$ of $S$, with $g \subset G$, and a fixed $\alpha$ in $\hat{G}$ for which $\Gamma=$ $\left\{t \alpha \cdot \mu_{x}: t \in T, x \in G\right\}$ where $\mu$ is Haar measure on $g, \mu_{x}$ its translate to $x g$. If $\Gamma$ is not circular then in addition to $g, G$ and $\alpha$ we have an integer $n \geqq 1$ and $a \varphi$ in $g^{\perp} \subset \hat{G}$ for which $\Gamma=\left\{\varphi(x)^{1 / n} \alpha \cdot \mu_{x}: x \in G\right\}$ where $\varphi(x)^{1 / n}$ runs over all $n$th roots of $\varphi(x)$. Conversely any such set of measures forms a subgroup of $\tilde{\widetilde{S}}$.

There remains only the last point which is fairly obvious in the circular case. In the non-circular case any subset $\Gamma$ of the type described is algebraically a group, and one need only verify its closure. But if $\varphi\left(x_{\delta}\right)^{1 / n} \alpha \cdot \mu_{x_{\delta}} \rightarrow \nu$ then by virtue of the compactness of $G$ we can find a confinal subnet for which $x_{\delta^{\prime}} \rightarrow x$, an element of $G$, whence $\alpha \cdot \mu_{x_{\delta^{\prime}}} \rightarrow$ $\alpha \cdot \mu_{x}$; since $\varphi\left(x_{\delta^{\prime}}\right) \rightarrow \varphi(x)$ some $n$th root $\varphi(x)^{1 / n}$ of $\varphi(x)$ is a cluster point of $\varphi\left(x_{\delta^{\prime}}\right)^{1 / n}$ and $\varphi(x)^{1 / n} \alpha \cdot \mu_{x}$ is thus a cluster point of our convergent net, hence $=\nu$ and $\nu \in \Gamma$.

REMARK 4.3. The first portion of our proof identifies the idempotents in $\tilde{\widetilde{S}}$ when $S$ is a compact non-abelian group. For the argument shows $\mu^{2}=\mu \neq 0 \Rightarrow|\mu|^{2}=|\mu|$, so that $|\mu|$ is Haar measure of a subgroup $g$, while $\rho_{\mu}$ again appears as a multiplicative character of $g$.

4.4. It may be worthwhile to note the analogue for $\widetilde{\widetilde{S}}$ of Theorem 3.2: for $S$ abelian and $\mu_{0} \in \widetilde{S}, N^{-1} \sum_{n=1}^{N} \mu_{0}^{n} \rightarrow 0$ unless there is an $\alpha$ in $C(S)$ which is unimodular and multiplicative on $S_{\left|\mu_{0}\right|}$ (the subsemigroup of $S$ generated by carrier $\left.\left|\mu_{0}\right|\right)$ satisfying $\mu_{0}=\alpha \cdot\left|\mu_{0}\right|$, in which case $N^{-1} \sum_{n=1}^{N} \mu_{0}^{n} \rightarrow \alpha \cdot\left(\right.$ Haar measure on the least ideal of $\left.S_{\left|\mu_{0}\right|}\right)$. For if $\Sigma$ is the subsemigroup of $\tilde{\widetilde{S}}$ generated by $\mu_{0}$ then as before $N^{-1} \sum_{n=1}^{N} \mu_{0}^{n} \rightarrow \nu$, the unique element of the least ideal of the semigroup $\mathscr{C}(\Sigma)$. Clearly $\nu=0$ if $0 \in \mathscr{C}(\Sigma)$; if $0 \notin \mathscr{C}(\Sigma)$ then

$$
\mu \in \mathscr{C}(\Sigma) \Rightarrow\|\mu\|=1 \text {, }
$$

and

(4.42) the least ideal $\mathscr{J}$ of $\Sigma$ is a non-circular subgroup of $\tilde{\tilde{S}}$ as in 4.2 with $n=1$.

For otherwise, in each case, we may conclude that $0 \in \mathscr{C}(\Sigma)$. Consequently $\mathscr{F}=\left\{\beta \cdot \eta_{x}: x \in G\right\}$ where $\beta \in \hat{G}, G$ is a subgroup of $S$ and $\eta$ is Haar measure of a further subgroup.

But just as in the proof of $4.2, \mu \rightarrow|\mu|$ is an algebraic homomorphism of $\mathscr{C}(\Sigma)$ into $\tilde{S}$. Moreover the image of $\mathscr{J}=\left\{\eta_{x}: x \in G\right\}$ is closed and is easily seen to be the least ideal of the closure $\Sigma_{1}$ of the image of $\Sigma$. Thus by Theorem 3.1, G is the least ideal of $S_{1}=$ carrier $\Sigma_{1}$, 
and as in theorem 3.5 we can extend $\beta$ to a continuous unimodular multiplicative function $\beta_{1}$ on $S_{1}$ by setting $\beta_{1}(x)=\beta(x e), x \in S_{1}$, where $e$ is the identity of the least ideal $G$ of $S_{1}$. Since $\beta_{1}^{-1}$ is multiplicative on $S_{1}$ and all $\mu$ in $\Sigma$ vanish on all Borel subsets of $S_{1}^{\prime}, \mu \rightarrow \beta_{1}^{-1} \cdot \mu$ is a homomorphism on $\Sigma$ which in particular maps $\mathscr{I}$ into $\tilde{S}$. As a consequence it must map all of $\Sigma$ into $\widetilde{S}$ : for if $\nu=\beta_{1}^{-1} \cdot \mu$ is the image of $\mu \in \Sigma$ then, since $(\beta \cdot \eta) \mu \in \mathscr{S}$ we have $\eta \nu \in \widetilde{S}$ whence $1=\eta \nu(1)=$ $\eta(1) \nu(1)=\nu(1)$, so $\|\nu\|=1, \nu \geqq 0$. Evidently then $\beta_{1}^{-1} \cdot \mu=|\mu|$. In particular $\mu_{0}=\beta_{1}\left|\mu_{0}\right|$ and we may take $\alpha$ as any continuous extension to all of $S$ of $\beta_{1}$. Finally if such an $\alpha$ is available then

$$
\alpha \cdot\left|\mu_{0}\right|^{n}=\left(\alpha \cdot\left|\mu_{0}\right|\right)^{n} \text { so } N^{-1} \sum_{n=1}^{N} \mu_{0}^{n}=\alpha \cdot\left(N^{-1} \sum_{n=1}^{N}\left|\mu_{0}\right|^{n}\right)
$$

and the final assertion follows from theorem 3.2.

5. Application to $C_{0}(\mathscr{G})^{*}$. Let $\mathscr{G}$ be a locally compact abelian group and $C_{0}(\mathscr{G})$ the Banach space of continuous functions vanishing at $\infty$, so that $C_{0}(\mathscr{C})^{*}$ consists of the finite regular Borel measures on $\mathscr{G}$. Uniform continuity of each element of $C_{0}(\mathscr{G})$ allows one to define convolution just as in $\S 1$, and $C_{0}(\mathscr{C})^{*}$ is easily seen to form an abelian semigroup. However, the natural choice of the $\omega^{*}$ topology of $C_{0}(\mathscr{G})^{*}$ will not yield the unit ball a topological semigroup ${ }^{3}$; rather it is the topology of pointwise convergence of Fourier-Stieltjes transforms (in which $\mu_{\delta} \rightarrow \mu \Leftrightarrow \hat{\mu}_{\delta}(\alpha) \rightarrow \hat{\mu}(\alpha)$ for each $\left.\alpha \in \hat{\mathscr{C}}\right)$ which does, and it is this topology we shall adopt.

The possibility of applying our previous results to the (topological) semigroup we thus obtain from the unit ball of $C_{0}(\mathscr{G})^{*}$ arises from two facts, both due to Eberlein [3]. Let $\mathscr{G}^{*}$ be the almost periodic compactification ${ }^{4}$ of $\mathscr{G}$. Then as Eberlein has noted there is an isometric imbedding of $C_{0}(\mathscr{G})^{*}$ into $C\left(\mathscr{G}^{*}\right)^{*}:$ for $\mu \in C_{0}(\mathscr{G})^{*}$ let $\mu^{\prime}(f)=\int f(x) \mu(d x)$ for $f$ almost periodic on $\mathscr{C}$. Since the almost periodic functions on $\mathscr{G}$ are isometrically isomorphic to $C\left(\mathscr{C}^{*}\right)$ we obtain $\mu^{\prime} \in C\left(\mathscr{G}^{*}\right)^{*}$. The clearly linear map $\mu \rightarrow \mu^{\prime}$ then preserves norms by the following argument: select a compact $K \subset \mathscr{G}$ for which $|\mu|\left(K^{\prime}\right)<\varepsilon$ and an element $f$ of the unit ball of $C_{0}(\mathscr{G})$ for which $\left|\int f(x) \mu(d x)\right| \geqq\|\mu\|-\varepsilon$, so that $\left|\int_{K} f(x) \mu(d x)\right| \geqq\|\mu\|-2 \varepsilon$. Since $\mathscr{G}$ has sufficiently many characters the map of $\mathscr{G}$ into $\mathscr{G}^{*}$ is one-to-one and thus a homeomorphism on $K$. Consequently ${ }^{5}$ we can find an $F$ in the unit ball of $C\left(\mathscr{G}^{*}\right)$ which extends

3 For example take $\mu_{n}=$ mass 1 at the integer $n \in R$; then $\mu_{n} \rightarrow 0$, and $\mu_{-n} \rightarrow 0$ in the $\omega^{*}$ topology of $C_{0}(R)^{*}$ as $n \rightarrow+\infty$ while $\mu_{n} \mu_{-n}=\mu_{0}$.

4 It will be convenient to view $\mathscr{C}$ as a dense algebraic subgroup of $\mathscr{C}^{*}$, and the almost periodic functions on $\mathscr{C}$ as the restrictions, to $\mathscr{C}$, of elements of $C\left(\mathscr{C}^{*}\right)$, cf. [6, 9].

5 We can simply extend the real and imaginary parts of $f \mid K$ separately by Urysohn's lemma to obtain an extension $F^{\prime \prime}$, and set $F(x)=F^{\prime \prime}(x) \cdot\left(1 \wedge\left|F^{\prime \prime}(x)\right|^{-1}\right)(=0$ of course if $\left.F^{\prime \prime}(x)=0\right)$. 
$f \mid K$, a continuous function on the compact subset $K$ of $\mathscr{C}^{*}$. Then we have

$$
\left|\mu^{\prime}(F)\right|=\left|\int_{\mathscr{G}} F(x) \mu(d x)\right| \geqq\left|\int_{K} f(x) \mu(d x)\right|-\varepsilon \geqq\|\mu\|-3 \varepsilon,
$$

and $\left\|\mu^{\prime}\right\| \geqq\|\mu\|-3 \varepsilon$. Evidently $\left\|\mu^{\prime}\right\| \leqq\|\mu\|$, so $\mu \rightarrow \mu^{\prime}$ is an isometry. Moreover it is clear that since both $\mathscr{G}$ and $\mathscr{G}^{*}$ have the same algebraic group of characters, the underlying group of $\hat{\mathscr{G}}$, we may write $\hat{\mu}=\hat{\mu}^{\prime}$ since both of these Fourier-Stieltjes transforms coincide as functions on the set $\hat{\mathscr{G}}$, and thus (since for measures $\mu, \nu$ on either group $\left(\mu_{\nu}\right)^{\wedge}$ $=\hat{\mu} \cdot \hat{\nu})(\mu \nu)^{\prime}=\mu^{\prime} \nu^{\prime}$. Consequently the map $\mu \rightarrow \mu^{\prime}$ is an algebraic isomorphism of the semigroup formed by the ball of $C_{0}(\mathscr{G})^{*}$ into that formed by the ball of $C\left(\mathscr{G}^{*}\right)^{*}, \tilde{\tilde{G}}^{*}$. Further, our choice of topology is just that which makes the map topological as well.

The second and crucial fact for our application which we obtain from Eberlein is the following corollary of the main result ${ }^{6}$ of [3]: Consider $C_{0}(\mathscr{G})^{*}$ as imbeded in $C\left(\mathscr{G}^{*}\right)^{*}$. Then its elements are just those measures $\mu$ on $\mathscr{G}^{*}$ with $\hat{\mu}$ continuous on $\hat{\mathscr{G}}$. Thus we can easily identify the range of $\mu \rightarrow \mu^{\prime}$.

Suppose then we are given a non-trivial closed subgroup $\Gamma$ of the unit ball of $C_{0}(\mathscr{C})^{*}$, and let $\Gamma_{0}$ be its isomorphic image in $\tilde{\mathscr{C}}^{*}$. Then $\Gamma_{0}^{-}$is a subgroup of $\tilde{\mathscr{G}}^{*}$ and thus by Theorem 4.2 each of its elements is of the form $t \alpha \cdot \eta_{x}$, where $t \in T, \alpha$ is a character of a subgroup $G_{0}$ of $\mathscr{C}^{*}, \eta$ is Haar measure of a further subgroup $g_{0}$ of $G_{0}$ and $\eta_{x}$ the translate of $\eta$ to the coset $x g_{0} \subset G_{0}$; indeed since each character of $G_{0}$ extends to one of $\mathscr{C}^{*}$, we shall take $\alpha \in \mathscr{G}^{* \wedge}$, i.e. as a character of $\mathscr{C}$. But the identity $\alpha \cdot \eta$ of $\Gamma_{0}^{-}$was already present in $\Gamma_{0}$ and thus has a continuous Fourier-Stieltjes transform on $\hat{\mathscr{G}}$, whence $\hat{\eta}$ is continuous on $\hat{\mathscr{G}}$. Since $\hat{\eta}=\varphi_{g_{0}^{+}}$, the characteristic function of the subgroup $g_{0}^{\perp}$ of $\mathscr{G}^{* \wedge}\left(=\hat{\mathscr{C}}\right.$ in the discrete topology) orthogonal to $g_{0}$, we obtain the fact that $g_{0}^{\perp}$ is an open and closed subgroup of $\hat{\mathscr{G}}$, and $\hat{\mathscr{G}} \mid g_{0}^{\perp}$ is discrete. But $\hat{\mathscr{S}} \mid g_{0}^{\perp}$ is the character group of that subgroup $g$ of $\mathscr{C}$ orthogonal to $g_{0}^{\perp}$; consequently $g$ is a compact subgroup of $\mathscr{G}$. If $\mu$ denotes its Haar measure then $\hat{\mu}=\varphi_{g^{+}}=\varphi_{g_{0}^{+}}=\hat{\eta}$ so $\eta=\mu^{\prime}$ by the one-to-one-ness of the Fourier-Stieltjes transformation.

Now consider a general element $t \alpha \cdot \eta_{x}$ of $\Gamma_{0}$. The fact that its

${ }^{6} \quad$ Specifically Eberlein's result may be stated as follows: for $\mu \in C\left(\mathscr{C}^{*}\right), \hat{\mu} \in L_{\infty}(\hat{\mathscr{C}})$ (in the usual sense) implies there is a $\vee$ in $C_{0}(\mathscr{C})^{*}$ for which $\hat{\vee}$ coincides with $\hat{\mu}$ in $L_{\infty}(\mathscr{C})$. Since here our $\hat{\mu}$ is continuous, as $\hat{\nu}$ must be, we obtain $\hat{\mu}=\hat{\vee}$ as functions and thus $\mu=v^{\prime}$ by the (one-to-one)-ness of the Fourier-Stieltjes transformation. 
transform is continuous implies $\left(\eta_{x}\right)^{\wedge}$ is continuous while

$$
\left(\eta_{x}\right)^{\wedge}(\beta)=\beta(x) \hat{\eta}(\beta)=\beta(x) \varphi_{g_{0}^{+}}(\beta) ;
$$

thus as a function of $\beta, \beta(x)$ is continuous on the open subset $g_{0}^{+}$of $\hat{\mathscr{G}}$, hence on all of $\hat{\mathscr{G}}$. By duality we then have a $y$ in $\mathscr{G}$ for which $\beta(x)=\beta(y)$, all $\beta$, and we may identify $x$ as an element of $\mathscr{C} \cap G_{0}$. Conversely each $x$ in $\mathscr{G} \cap G_{0}$ gives rise to elements of $\Gamma_{0}^{-}$which already lie in $\Gamma_{0}$ (for such measures lie in the image of $C_{0}(\mathscr{G})^{*}$ in which $\Gamma_{0}$ is relatively closed by hypothesis) ; thus $\Gamma_{0}$ consists of just those elements $t \alpha \cdot \eta_{x}$ of $\Gamma_{0}^{-}$arising from $x$ 's in $\mathscr{C} \cap G_{0}=G$, algebraically a subgroup of $\mathscr{G}$. But clearly $G$ is closed in $\mathscr{C}$, and is thus a subgroup of $\mathscr{G}$, since the map $\mathscr{G} \rightarrow \mathscr{G}^{*}$ is continuous.

Finally it is clear that if $\Gamma$ (and thus $\Gamma_{0}$ ) is circular so is $\Gamma_{0}^{-}$; conversely if $\Gamma_{0}^{-}$is circular then $T \alpha \cdot \eta \subset \Gamma_{0}^{-}$and thus $T \alpha \cdot \eta \subset \Gamma_{0}$, whence $\Gamma_{0}$ and $\Gamma$ are circular. We have proved

THEOREM 5.1 Let $\mathscr{G}$ be a locally compact abelian group and let $C_{0}(\mathscr{G})^{*}$ be topologized by pointwise convergence of Fourier-Stieltjes transforms. Then any closed convolution subgroup $\Gamma$ of the unit ball of $C_{0}(\mathscr{G})^{*}$ is determined as in Theorem 4.2 where $g$ is a compact subgroup of $\mathscr{G}, G$ is a closed subgroup, and $\alpha$ and $\varphi$ may be taken as elements of $\hat{\mathscr{G}}$.

5.2. It should be noted that the convolution semigroup formed by the ball of $C_{0}(\mathscr{G})^{*}$, although not compact, shares some properties of compact semigroups: the closure of an algebraic subgroup is again a group, indeed a topological group in the relative topology (thus the last applies to an algebraic subgroup). For if $\Gamma$ is an algebraic subgroup its image $\Gamma_{0}$ in $C\left(\mathscr{G}^{*}\right)^{*}$ is an algebraic group, so that $\Gamma_{0}^{-}$is a compact topological group. But of course $\Gamma^{-}$is just the preimage of the intersection of $\Gamma_{0}^{-}$with the image of $C_{0}(\mathscr{G})^{*}$.

Finally suppose $\Gamma$ is a non-trivial algebraic subgroup of the ball of $C_{0}(\mathscr{G})^{*}$ which in addition is $\omega^{*}$ closed (compact). Then $\Gamma$ is a closed subgroup as described in Theorem 5.1 with $G$ a compact subgroup of $\mathscr{G}$ (and conversely). For, changing our notation, let $G$ denote the subgroup of $\mathscr{G}$ produced via Theorem 5.1 for $\Gamma^{-}$. Then the set $H$ of $x$ in $G$ corresponding to elements $t \alpha \cdot \mu_{x}$ in $\Gamma$ forms a dense algebraic subgroup of $G$, as is easily seen. If $G$ is not compact then we have a net $\left\{x_{\delta}\right\} \subset H$ which tends to $\infty$, so that the corresponding net of measures $\left\{t_{\delta} \alpha \cdot \mu_{x_{\delta}}\right\}$ tends to 0 in the $\omega^{*}$ topology ( $g$ being compact). But this implies $0 \in \Gamma$, which is clearly nonsense.

Consequently $G$ is compact and, since the elements of $\Gamma$ all vanish off $G$, the $\omega^{*}$ topology on $\Gamma$ reduces to the topology of pointwise 
convergence of Fourier-Stieltjes transforms (by virtue of the StoneWeierstrass theorem and the existence of sufficiently many characters of $\mathscr{C})$. Therefore the image of $\Gamma$ in $C\left(\mathscr{C}^{*}\right)^{*}$ is compact and closed, whence $\Gamma^{-}=\Gamma^{-}$.

\section{REFERENCES}

1. L. Alaoglu and G. Birkhoff, General ergodic theorems, Ann. of Math. (2) 41 (1940), 293-309.

2. R. F. Arens and J. L. Kelley, Characterizations of the space of continuous functions over a compact Hausdorff space, Trans. Amer. Math. Soc., 62 (1947), 499-508.

3. W. F. Eberlein, Characterizatıons of Fourier-Stieltjes transforms, Duke Math. J., 22 (1955), 465-468.

4. B. Gelbaum, G. K. Kalisch, and J. M. H. Olmsted, On the imbedding of topological semigroups and integral domains, Proc. Amer. Math. Soc., 2 (1951), 807-821.

5. P. R. Halmos, Measure theory, New York, 1950.

6. L. H. Loomis, An introduction to abstract harmonic analysis, New York, 1953.

7. K. Numakura, On bicompact semigroups, Math. J., Okayama University, 1 (1952), 99 -108 .

8. A. D. Wallace, The structure of topological semigroups, Bull. Amer. Math. Soc., 61 (1955), 95-112.

9. A. Weil, L'Integration dans les groupes topologiques ol ses applications, Actualites Seientifiques et Industrielles, no. 869, Paris, 1940.

10. J. G. Wendel, Haar measure and the semigroup of measures on a compact group, Proc. Amer. Math. Soc., 5 (1954), 923-929.

UNIVERSITY OF NOTRE DAME 



\section{PACIFIC JOURNAL OF MATHEMATICS}

\section{EDITORS}

\section{David Gilbarg}

Stanford University

Stanford, California

R. A. Beaumont

University of Washington

Seattle 5 , Washington
A. L. Whiteman

University of Southern California

Los Angeles 7, California

L. J. PAIGE

University of California

Los Angeles 24, California

\section{ASSOCIATE EDITORS}
E. F. BECKENBACH
C. E. BURGESS
E. HEWITT
A. HORN

\author{
V. GANAPATHY IYER \\ R. D. JAMES \\ M. S. KNEBELMAN \\ L. NACHBIN
}
I. NIVEN
E. G. STRAUS
T. G. OSTROM
H. L. ROYDEN
G. SZEKERES
M. M. SCHIFFER
F. WOLF
K. YOSIDA

\section{SUPPORTING INSTITUTIONS}

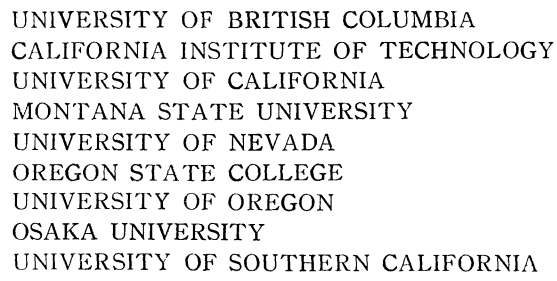

UNIVERSITY OF BRITISH COLUMBIA CALIFORNIA INSTITUTE OF TECHNOLOGY UNIVERSITY OF CALIFORNIA MONTANA STATE UNIVERSITY UNIVERSITY OF NEVADA OREGON STATE COLLEGE UNIVERSITY OF OREGON OSAKA UNIVERSITY UNIVERSITY OF SOUTHERN CALIFORNIA

STANFORD UNIVERSITY

UNIVERSITY OF TOKYO

UNIVERSITY OF UTAH

WASHINGTON STATE COLLEGE

UNIVERSITY OF WASHINGTON

AMERICAN MATHEMATICAL SOCIETY CALIFORNIA RESEARCH CORPORATION HUGHES AIRCRAFT COMPANY SPACE TECHNOLOGY LABORATORIES

Printed in Japan by Kokusai Bunken Insatsusha (International Academic Printing Co., Ltd.), Tokyo, Japan 


\section{Pacific Journal of Mathematics}

\section{Vol. 9, No. 1 \\ May, 1959}

Julius Rubin Blum and Murray Rosenblatt, On the structure of infinitely

divisible distributions . ............................. 1

Robert Geroge Buschman, Asymptotic expressions for

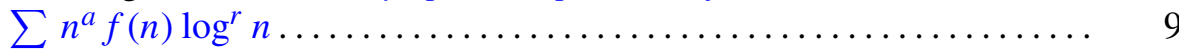

Eckford Cohen, A class of residue systems $(\bmod r)$ and related arithmetical

functions. I. A generalization of Möbius inversion .............. 13

Paul F. Conrad, Non-abelian ordered groups ................... 25

Richard Henry Crowell, On the van Kampen theorem............... 43

Irving Leonard Glicksberg, Convolution semigroups of measures ........ 51

Seymour Goldberg, Linear operators and their conjugates ............ 69

Olof Hanner, Mean play of sums of positional games .............. 81

Erhard Heinz, On one-to-one harmonic mappings ................ 101

John Rolfe Isbell, On finite-dimensional uniform spaces . . ........... 107

Erwin Kreyszig and John Todd, On the radius of univalence of the function

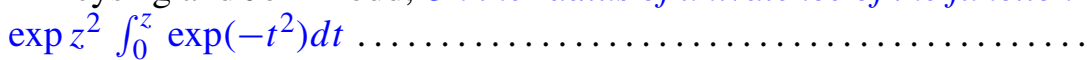

Roger Conant Lyndon, An interpolation theorem in the predicate

calculus......................................... 129

Roger Conant Lyndon, Properties preserved under homomorphism ........ 143

Roger Conant Lyndon, Properties preserved in subdirect products ....... 155

Robert Osserman, A lemma on analytic curves ................ 165

R. S. Phillips, On a theorem due to Sz.-Nagy..................... 169

Richard Scott Pierce, A generalization of atomic Boolean algebras ....... 175

J. B. Roberts, Analytic continuation of meromorphic functions in valued fields................................. 183

Walter Rudin, Idempotent measures on Abelian groups ................ 195

M. Schiffer, Fredholm eigen values of multiply-connected domains ........ 211

V. N. Singh, A note on the computation of Alder's polynomials ......... 271

Maurice Sion, On integration of 1-forms ...................... 277

Elbert A. Walker, Subdirect sums and infinite Abelian groups........... 287

John W. Woll, Homogeneous stochastic processes . .................. 293 